\title{
Uma tradução adaptada do texto sobre algumas refutações da existência de elétrons no núcleo, propostas por Bethe e Bacher
}

\author{
An adapted Portuguese translation of the text proposed by Bethe and Bacher: Disproof of the \\ Existence of Electrons in Nuclei \\ Gilberto Orengo* \\ Centro Universitário Franciscano, Santa Maria, RS, Brasil
}

Recebido em 21 de Novembro, 2016. Aceito em 16 de Dezembro, 2016.

\begin{abstract}
Neste artigo, é apresentada uma tradução adaptada de um importante texto de Bethe e Bacher, publicado em 1936, no qual são discutidas cinco razões para a não existência de elétrons no núcleo atômico.
\end{abstract}

Palavras-chave: decaimento beta, spin nuclear, momento de dipolo magnético nuclear, dimensões nucleares.

An adapted Portuguese translation of a text by Bethe and Bacher published in 1873 is offered, which are discussed a disproof of the existence of electrons in the atomic nucleus.

Keywords: beta decay, nuclear spins, nuclear magnetic dipole moment, nucleus size.

\section{Introdução}

Este artigo resgata o texto de Bethe e Bacher 1 , p. 184], referente às principais refutações da existência de elétrons no núcleo, cujo título do capítulo é $\beta$ Disintegration and Nuclear Forces, e do parágrafo é Disproof of the Existence of Electrons in Nuclei. Essa revisão sobre Física Nuclear foi publicada em 1936, e serve como base para este texto, numa tradução comentada e adaptada às atuais teorias e suposições a respeito do núcleo. O núcleo atômico é constituído por núcleons, denominação dada aos nêutrons $(n)$ e prótons $(p)$, e por partículas mediadoras das interações nucleares, entre as quais há os mésons- $\pi$ responsáveis pela coesão do núcleo - por intermédio das interações da força forte entre os núcleons, e os bósons $W^{+}, W^{-}$e $Z^{0}$, responsáveis pelas interações fracas nos decaimentos beta $\left(\beta^{-}\right.$e $\left.\beta^{+}\right)$e captura eletrônica (E.C. ou $\varepsilon)$ [2 4].

Um núcleo energeticamente instável, para se estabilizar, tende a emitir partículas, que podem ser

*Endereço de correspondência: g.orengo@gmail.com alfa $(\alpha), \beta$ ou $\varepsilon$, e neutrinos $(\nu)$, associadas ou não à emissão de radiação gama $(\gamma)$. No caso do decaimento beta, há emissão de um elétron $\left(e^{-}\right)$ou pósitron $\left(e^{+}\right)$, acompanhado de um neutrino, conforme exemplifica a seguinte representação de transmutação, respectivamente, para o decaimento $\beta^{-} \mathrm{e}$ $\beta^{+}$,

$$
\begin{aligned}
n & \rightarrow p+e^{-}+\bar{\nu}_{e}, \\
p & \rightarrow n+e^{+}+\nu_{e},
\end{aligned}
$$

em que $\nu_{e}$ é o neutrino do elétron e $\bar{\nu}_{e}$ é o seu antineutrino. Desse modo, a questão nessa emissão de elétrons e pósitrons é se eles "existiam" no núcleo antes de surgirem no seu exterior. Isto é, no caso do decaimento $\beta^{-}$, o nêutron, ao se transmutar em próton, antineutrino do elétron e elétron, este permanece, por algum tempo, no núcleo?

O núcleo, nos primórdios da Física Nuclear, por hipótese, era composto por elétrons e prótons. O número destes era igual ao número de massa $\mathrm{A}$, porque somente eles contribuíam para a massa do núcleo, enquanto que os $\mathrm{A}-\mathrm{Z}$ elétrons neutralizavam a carga de $\mathrm{A}-\mathrm{Z}$ prótons, que resultava na carga 
elétrica Z do núcleo [1, p. 90]. O próprio Rutherford [5. p. 584] lançou a hipótese de elétrons no núcleo para explicar o decaimento alfa. Ele supôs que a partícula alfa era neutra, devido aos seus dois elétrons, e orbitava ao redor do núcleo por conta de uma força central, em uma órbita quantizada. Ao abandonar o núcleo, a partícula alfa tinha os seus dois elétrons "roubados", que permaneciam no núcleo. No entanto, Gamow refutou essa suposição em seu trabalho a respeito da teoria quântica para o núcleo atômico [6, p. 205]. Desta forma, essa hipótese logo foi descartada, porque não acompanhava os resultados experimentais. Diante disso, são apresentadas algumas razões para a não existência de elétrons no núcleo.

\section{A tradução adaptada}

A seguir, é apresentada a tradução adaptada e comentada. A padronização adotada para a escrita é conforme a tipografia: a tradução do texto original, com adaptações somente de linguagem está em itálico, e os comentários do autor estão na sequência de cada tradução, em fonte igual ao restante do texto, bem como em notas de rodapé, indicadas pelas letras N.T., que representam a abreviatura de Nota do Tradutor.

\section{As principais refutações da existência de elétrons no núcleo}

Atualmente, acredita-se que não há elétrons no interior do núcleo atômico. As principais razões são as seguintes:

\section{A descrição estatistica dos núcleos}

Os núcleos com número de massa par geralmente obedecem a estatística de Bose-Einstein, enquanto que os com número de massa ímpar obedecem a estatística de Fermi-Dirac. Esse resultado é esperad 1

${ }^{1}$ N.T. Neste ponto, os autores remetem à p. 89, da Ref. [1], para uma fundamentação sobre o assunto. se o núcleo contém somente nêutrons e prótons. Se for considerada, como exemplo, a troca de um nêutron por um próton mais um elétron, acarretará no aumento de uma partícula elementan o que alteraria a estatística de Bose para Fermi e vice-versa.

Todas as partículas na natureza obedecem ou a Estatística de Fermi-Dirac ou a de Bose-Einstein. A estatística "quântica" de Bose-Einstein determina a distribuição dos estados energéticos de partículas idênticas e indistinguíveis, com spin inteiro, conhecidas como bósons. São exemplos o fóton, o deutério, a partícula alfa, entre outros. Já a estatística de Fermi-Dirac, também é uma estatística quântica, e rege a distribuição dos estados energéticos das partículas indistinguíveis, com spin semi-inteiro, os férmions. Os elétrons (positivo e negativo) e prótons são partículas fermiônicas, assim como os nêutrons, neutrinos, o trítio, entre outros. Mesmo assim, tanto Fermi-Dirac como Bose-Einstein tornamse a estatística de Maxwell-Boltzmann quando consideradas as altas temperaturas ou baixas concentrações [7].

Deste modo, na Tabela 11, são apresentados dados nucleares para alguns nuclídeo $3^{3}$, a fim de exemplificar e corroborar as afirmações anteriores, as quais indicam que os elétrons não existem no núcleo. Embora sejam apresentados somente seis nuclídeos, numa pesquisa na base de dados da Agência Internacional de Energia Atômica (IAEA) [8], observa-se que essa classificação se mantém para os demais nuclídeos.

\footnotetext{
${ }^{2}$ N.T. Os nêutrons e prótons, hoje, não são mais considerados partículas elementares, mas sim, constituídos de quarks. O nêutron é formado por um quark up $(u)$ e dois quarks down $(d)$, e o próton por dois quarks up e um quark down, cujas cargas elétricas são $q_{u}=+\frac{2}{3} e$ e $q_{d}=-\frac{1}{3} e$, em que $e$ é o valor da carga elétrica do elétron.

${ }^{3}$ N.T. Nuclídeo é uma espécie nuclear caracterizada pelo seu número atômico e número de massa.
}

Tabela 1: Dados nucleares para alguns nuclídeos: aqueles com A-par têm spin inteiro, e os com A-ímpar têm spin semi-inteiro, com suas respectivas paridades e descrições estatísticas. $\mathrm{O}$ momento de dipolo $(\mu)$ dos nuclídeos é apresentado em termos de $\mu_{N}$ (o magnéton nuclear) [8].

\begin{tabular}{lcccccc}
\hline Nuclídeo & $\mathrm{Z}$ & $\mathrm{N}$ & $\mathrm{A}=\mathrm{Z}+\mathrm{N}$ & Spin/Paridade $\left(J^{\pi}\right)$ & $\mu\left(\mu_{N}\right)$ & Estatística \\
\hline Ferro (Fe) & 26 & 30 & 56 & $0^{+}$ & $+1,02$ & \\
Cádmio (Cd) & 48 & 62 & 110 & $0^{+}$ & $+0,81$ & Bose- \\
Bromo (Br) & 35 & 43 & 78 & $1^{+}$ & $+0,13$ & Einstein \\
Césio (Cs) & 55 & 79 & 134 & $4^{+}$ & $+2,9937$ & \\
\hline Potássio (K) & 19 & 22 & 41 & $(3 / 2)^{+}$ & $+0,2148701$ & Fermi- \\
Cobalto (Co) & 27 & 32 & 59 & $(7 / 2)^{-}$ & $+4,627$ & Dirac \\
\hline
\end{tabular}




\section{O spin nuclear}

Um argumento equivalente é válido para os spins nucleares, que são inteiros ou semi-inteiros, conforme o número de massa seja par ou impar

O spin nuclear, ou momento angular total dos constituintes do núcleo, é definido como a soma vetorial dos momentos angulares orbital e intrínseco, de todos os núcleons, no estado fundamental [2].

O preenchimento dos níveis energéticos, no núcleo, segue a regra do pareamento, diferente do que ocorre com os elétrons, na eletrosfera, os quais, além de também obedecerem o Princípio de exclusão de Pauling, são distribuídos de acordo com a regra de Hund [3]. Desta forma, os spins emparelhados, no caso dos núcleos Z-par N-par (A-par), resultarão num spin nulo para o núcleo. Nos núcleos Z-ímpar N-ímpar (A-par), os spins desemparelhados para prótons e nêutrons fornecerão um spin inteiro não nulo ou nulo. Já para os núcleos com A-ímpar, o spin nuclear será indicado pelo núcleon desemparelhado, conforme a sua "posição" nos níveis de energia propostos pelo Modelo de Camadas, para os estados fundamentais [2,9].

O argumento, para refutar a existência de elétrons no núcleo, segue o adotado no item 1 , isto é, núcleos com A-par têm spin inteiro, e os com A-ímpar possuem spin semi-inteiro, o que também é evidenciado na classificação apresentada na Tabela 1. Assim, se ocorrer o aumento de uma partícula de spin semiinteiro, no caso, o elétron, haverá uma troca no spin nuclear, que resulta num desacordo com os dados experimentais previstos e evidencia que o elétron não existe no núcleo.

\section{O momento de dipolo magnético nuclear}

Os momentos de dipolo magnético nuclear são todos da ordem do magnéton do próton eћ/2Mc, enquanto que deveriam ser da ordem do magnéton de Bohr eћ/2mc, se os elétrons existissem no núcle 5 .

O momento de dipolo magnético para o núcleo é medido em termos do magnéton nuclear, que é definido usando a massa do próton $\left(m_{p}\right)[2]$,

$$
\begin{aligned}
\mu_{N} & =g \frac{e}{2 m_{p}} S_{p} \approx 5,05 \times 10^{-27} \mathrm{~J} / \mathrm{T} \\
& =3,15 \times 10^{-14} \mathrm{MeV} / \mathrm{T}
\end{aligned}
$$

\footnotetext{
${ }^{4}$ N.T. Neste ponto, os autores remetem à p. 90, da Ref. [1, para uma fundamentação sobre o assunto.

${ }^{5}$ N.T. Nas equações, escritas no sistema de unidades CGS, temos: $e$ é a carga elementar; $M$ é a massa do próton; $\hbar=$ $h / 2 \pi$, em que $h$ é a constante de Planck; $c$ é a velocidade da luz no vácuo e $m$ é a massa do elétron.
}

em que $g=2$ é o fator de Landé $(g)^{6}$, é o valor da carga do elétron, e $S_{p}$ é o spin do próton, cujo valor é $\frac{1}{2} \hbar$. Para o elétron, o momento de dipolo magnético é avaliado pelo magnéton de Bohr:

$$
\begin{aligned}
\mu_{B} & =\frac{e \hbar}{2 m_{e}} \approx 9,27 \times 10^{-24} \mathrm{~J} / \mathrm{T} \\
& =5,79 \times 10^{-11} \mathrm{MeV} / \mathrm{T} .
\end{aligned}
$$

Comparando os dois valores obtém-se:

$$
\frac{\mu_{B}}{\mu_{N}} \approx 1835,6
$$

Já o valor do momento de dipolo magnético nuclear $\left(\mu_{N}\right)$ é, aproximadamente, 1800 vezes menor do que o do elétron, e, desta forma, evidencia-se que o elétron não pode estar presente no núcleo, pois ficará difícil justificar os pequenos valores do momento magnético dos núcleos. Desse modo, na Tabela 1 são apresentados os valores de momento de dipolo magnético para alguns nuclídeos, os quais são muito menores comparados com o magnéton de Bohr.

\section{O tamanho da função de onda para os elétrons}

O comprimento de onda de um elétron com uma energia cinética da ordem de $\mathrm{MV}^{7}$ (energia da maioria das partículas $\beta$ !) é muito maior do que o raio nuclear 8

O raio do núcleo vai de aproximadamente 1,6755× $10^{-15} \mathrm{~m}$, para a partícula- $\alpha$, até aproximadamente $5,8571 \times 10^{-15} \mathrm{~m}$ para o núcleo de urânio (U-238) 10 .

${ }^{6} \mathrm{O}$ fator de Landé é usado para investigar os aspectos fundamentais de estruturas baseadas em spin e é uma quantidade adimensional caracterizada pela relação entre o momento magnético e o raio giromagnético de uma partícula, imersa num campo magnético fraco. O campo cancela a degeneração dada pela uniformização do momento angular orbital, e, assim, o fator de Landé é obtido a partir do cálculo de perturbações, em primeira ordem. Valores do fator de Landé diferentes de 2,0 indicam que a partícula, provavelmente, possui estrutura interna, e, portanto, não é classificada como "partícula elementar". Como exemplo, tem-se, respectivamente, para os elétrons, múons, nêutrons e prótons, valores de $g$ iguais a $-2,00232,-2,00233,-3,82608$ e $+5,58569$.

${ }^{7}$ N.T. MV é representado atualmente como MeV.

${ }^{8}$ N.T. Neste ponto, os autores remetem à p. 88 , da Ref. 1], para uma fundamentação sobre o assunto.

${ }^{9}$ N.T. Os autores remetem o leitor para uma página que fundamenta a discussão e na qual está indicado o intervalo do raio nuclear, à época, que ia de aproximadamente 2 ou $3 \times 10^{-15} \mathrm{~m}$, para a partícula- $\alpha$, até aproximadamente $9 \times 10^{-15} \mathrm{~m}$ para o núcleo de urânio [1 p. 88]. Na maioria dos livros sobre Física Nuclear, o raio nuclear pode ser estimado por $R=r_{0} \times A^{1 / 3}$, em que $r_{0} \approx 1,2 \times 10^{-15} \mathrm{~m} 2,4$. Uma atualização dessa expressão foi proposta por Angeli e Mari- 
Um aspecto interessante, sobre as dimensões nucleares, é que o volume de um núcleo é aproximadamente proporcional ao seu número de massa $\mathrm{A}$,

$$
\begin{aligned}
V & =\frac{4}{3} \pi R^{3}=\frac{4}{3} \pi\left(r_{0} \times A^{1 / 3}\right)^{3} \approx \frac{4}{3} \pi r_{0}^{3} A ; \\
\frac{V}{A} & \approx \frac{4}{3} \pi r_{0}^{3}=\text { constante },
\end{aligned}
$$

de modo que o volume por núcleons é praticamente o mesmo em cada núcleo.

Outra propriedade de importante implicação, para os propósitos deste estudo, é que o valor médio da energia de ligação por núcleon, para a grande maioria dos nuclídeos, é $8,0 \mathrm{MeV} /$ núcleon. Isto significa que, se for depositada uma energia de 8,0 MeV no interior do núcleo, então, é possível "libertar" um núcleon do estado ligado pela força forte [2].

Vamos usar essa propriedade juntamente com a hipótese de De Broglie, para investigar as dimensões nucleares. O físico francês, Louis De Broglie, em 1924, em sua tese de doutorado, formulou uma hipótese afirmando que, do ponto de vista quântico, uma onda pode ser associada com cada partícula (ou corpúsculo) [11, p. 46], cujo comprimento de onda reduzido $(\lambda)$ está relacionado com o seu momento linear por

$$
\lambda=\frac{\hbar}{p},
$$

em que $\hbar=h / 2 \pi$, com $h$ sendo a constante de Planck, e $p=m v$, o momento linear da partícula. Na hipótese de Broglie, os estados ligados requerem que

$$
2 \pi r=n \lambda \quad \Rightarrow \quad r=n \lambda
$$

tal que o $\lambda=\lambda / 2 \pi$ fornece indícios de um típico tamanho do raio nuclear. Se for adotada a energia de $8,0 \mathrm{MeV}$, que é a energia média de ligação de um núcleon, o seu comprimento de onda reduzido, para $n=1$, será

$$
\begin{aligned}
\lambda & =\frac{\hbar}{p}=\frac{\hbar}{\sqrt{2 m E_{K}}}=\frac{\hbar c}{\sqrt{2 m c^{2} E_{K}}} \\
& \cong 1,6 \mathrm{fm}=1,6 \times 10^{-15} \mathrm{~m},
\end{aligned}
$$

em que $\hbar c=197,326 \mathrm{MeVfm} ; m c^{2}=$ $938,918 \mathrm{MeV} 10$ e $E_{K}=8,0 \mathrm{MeV}$. Este resultado

nova (2013) que é $R=\left(r_{0}+r_{1} \times A^{-2 / 3}-r_{2} \times A^{-4 / 3}\right) A^{1 / 3}$, em que $r_{0}=0,9071(13) \mathrm{fm}=0,9071(13) \times 10^{-15} \mathrm{~m}$, $r_{1}=1,105(25) \mathrm{fm}$ e $r_{2}=-0,548(34) \mathrm{fm}$. Assim, para o He-4, tem-se $\mathrm{R} \approx 1,999 \mathrm{fm}$, e para o U-238, $\mathrm{R} \approx 5,797 \mathrm{fm}$. Os valores medidos, já mencionados, são respectivamente, $1,6755 \mathrm{fm}$ e $5,8571 \mathrm{fm} 10$.

${ }^{10}$ N.T. Foi adotada uma média entre os valores de $m c^{2}$ do próton $(938,272 \mathrm{MeV})$ e do nêutron $(939,565 \mathrm{MeV})$ 12. permite concluir que os prótons e nêutrons, como esperado, com essa energia, existem ou "residem" no núcleo, pois o valor associado de seus comprimentos de onda reduzido está em acordo com a dimensão nuclear e, desta forma, podem orbitar no núcleo.

A seguir, é apresentado o resultado quando se trata de elétrons, com essa mesma energia, característica do núcleo.

Para os elétrons, será considerado o momento linear dado por

$$
p=\frac{\sqrt{\left(E_{K}\right)^{2}-\left(m_{0} c^{2}\right)^{2}}}{c}
$$

que resulta num comprimento de onda reduzido

$$
\begin{aligned}
\lambda & =\frac{\hbar c}{\sqrt{\left(E_{K}\right)^{2}-\left(m_{0} c^{2}\right)^{2}}} \cong 24,7 \mathrm{fm} \\
& =24,7 \times 10^{-15} \mathrm{~m},
\end{aligned}
$$

com $m_{0} c^{2}=0,510 \mathrm{MeV}$ e $E_{K}=8,0 \mathrm{MeV}$. O resultado é uma forte evidência de que o elétron não pode estar presente no núcleo, porque o seu comprimento de onda associado é muito maior que as dimensões típicas de um núcleo atômico, e, assim, é difícil sustentar que o elétron orbitará numa região com as dimensões do núcleo. Se, por outro lado, forem considerados os valores de energia das partículas no decaimento- $\beta$, que são da ordem de pouco mais de $1 \mathrm{MeV}$, o resultado será ainda maior para o $\lambda$. Por exemplo, o Ba-147 possuiu uma energia média no decaimento $\beta^{-}$de $2,5 \mathrm{MeV}[8]$ e, portanto, tem um comprimento de onda reduzido da ordem de 80,6 fm. Este valor é ainda maior e corrobora a suposição da não existência do elétron no núcleo.

5. A impossibilidade de uma barreira de potencial para manter os elétrons no núcleo

Este argumento é o mais forte de todos e será discutido com mais detalhes.

Os núcleos que emitem partícula $\beta$ têm uma vida média de $1 / 50$ de segundo até aproximadamente $10^{8}$ anos. Deve ter, portanto, por hipótese, alguma força para manter as partículas $\beta$ no interior do núcleo, apesar de o fato dessa possuir energia suficiente para escapar. Deve-se, então, assumir que há uma barreira de potencial mantendo os elétrons no núcleo, em analogia com as partículas alfa $(\alpha)$. Mas existem três importantes razões contrárias a essa hipótese, as quais são discutidas a seguir.

(a) De acordo com o que já é conhecido, um núcleo atrai um elétron a qualquer distância. Isto 
certamente é verdade para grandes distâncias (pela força de Coulomb) e, para distâncias muito pequenas (assumindo que existam elétrons ligados no núcleo). A fim de fornecer uma barreira potencial para os elétrons, deveria haver uma forte repulsão em distâncias intermediárias, neste caso, algumas vezes a distância do raio do núcleo.

(b) Conforme a teoria relativística, é quase impossivel ter uma barreira de potencial que mantenha um elétron com alta energia aprisionado no núcleo. Para verificar isso, é suficiente considerar a equação de Schrödinger relativística (sem o spin $1^{11}$,

$$
\hbar^{2} c^{2} \Delta \psi+\left[(E-V)^{2}-m^{2} c^{4}\right] \psi=0,
$$

em que E é a energia total do elétron, isto é, sua energia cinética a uma distância infinita do núcleo mais $m c^{2}$. Essa equação tem uma solução de caráter exponencial somente se

$$
|E-V|<m c^{2} .
$$

Entretanto, é necessário que a solução seja do tipo exponencial, na região da barreira de potencial, porque somente neste caso essa barreira evita que as partículas escapem. Isto significa que a energia potencial $V$ no interior dessa região não deve diferir da energia total $E$ do elétron por mais do que $m c^{2}$. É óbvio que tal exigência é muito improvável de ser cumprida por um determinado potencial barreira, especialmente para tais núcleos, nos os quais a energia $E$ das partículas beta é muito grande. Por exemplo, no caso do Boro-12 (B $\left.{ }^{12}\right)$, a energia $E=24 m c^{2}$. Então, $V$ deveria estar entre 23 e $25 \mathrm{mc}^{2}$, que, de fato, é uma hipótese muito improváve ${ }^{12}$

(c) Certo de que a energia potencial $V$ tem um valor satisfeito, pela equação (13), seriam extremamente grandes as perturbações dos elétrons

\footnotetext{
${ }^{11}$ N.T. Atualmente, o Laplaciano $\Delta$ é representado por $\nabla^{2}$, sendo $\nabla$ o operador "diferencial" Nabla.

${ }^{12} O$ fato de as partículas beta provenientes de um determinado núcleo terem um espectro contínuo tornaria a situação insustentável, porque teríamos que assumir uma altura diferente da barreira de potencial para diferentes núcleos da mesma espécie, correspondendo a energia da partícula beta emitida. Mas a distribuição contínua de energia dos raios- $\beta$ não pode ser completamente entendida sem a presença de uma outra partícula: o neutrino.
}

ópticos devido a esse potencial, o que absolutamente contradiz o experimento. A suposição mais favorável é que $V=E$, no interior da barreira, para $r$ entre $R$ e $R+b$, em que $R$ é o raio nuclear, $b$ é a largura da barreira de potencial e $r$ é a distância entre o núcleo e o elétron. Neste caso, a solução da equação (12) é:

$$
\psi(r)=A \mathrm{e}^{-m c r / \hbar}, \text { para } R<r<R+b,
$$

em que A é uma constante. O tempo de vida médio é, então, similar a de um núcleo emitindo partículas alfa, ou seja,

$$
\tau=\left(\frac{R}{c}\right) e^{2 m c b / \hbar} .
$$

Usando $R=8 \times 10^{-15} \mathrm{~m}, c=3 \times 10^{8} \mathrm{~m} / \mathrm{s}, e$ assumindo um tempo de vida médio $\tau=1 \mathrm{~s}$, temos

$$
\begin{aligned}
\frac{2 m c b}{\hbar} & =\ln \left(4 \times 10^{22}\right)=52 \\
b & =26\left(\frac{\hbar}{m c}\right)=26 \lambda_{C},
\end{aligned}
$$

isto é, a largura da barreira de potencial deve ser muito maior do que o comprimento de onda Compton $\lambda_{C}=\hbar / m c$. Uma vez que o raio da camada $K$, de átomos pesados, é inferior a duas vezes o comprimento de onda Compton, a barreira de potencial proposta nesta hipótese mudaria a atuação do potencial sobre os elétrons da camada $K$, e até mesmo nos elétrons mais distantes, e teria um enorme impacto sobre as energias de todos esses elétrons. Desta maneira, é refutada completamente a hipótese de uma barreira de potencial mantendo os elétrons no núcleo.

Desta forma, após todas as considerações anteriores, somos forçados a assumir que os elétrons observados na desintegração beta não pré-existem no núcleo emissor. Supõe-se, então, que eles são formados no mesmo momento em que são realmente emitidos, sendo este o processo de formação que torna improvável a explicação dos tempos de vida longa dos núcleos emissores de beta.

$O$ processo de desintegração- $\beta$ não deve ser comparado com o da desintegração- $\alpha$, mas sim com a emissão de luz pelos átomos (ou núcleos). Esta comparação com a luz parece mais vantajosa para 
explicar o que se entende por não pré-existência e formação no momento da emissão. Ninguém diria que um átomo de hidrogênio, no terceiro estado excitado, contém o quanta de luz correspondente às linhas espectrais que ele pode emitir, isto é, a linha $H-\alpha$ e as duas primeiras linhas da série de Lyman (a segunda linha de Lyman pode ser emitida imediatamente, ou a primeira, após a emissão da $H-\alpha)$. Ainda assim, o átomo de hidrogênio é capaz de emitir quanta de luz destes, e é geralmente aceito que os quanta são produzidos no momento da sua emissão. A emissão de elétrons por núcleos é totalmente análoga, temos apenas que substituir "elétron" pelo "quantum de luz".

Nesse sentido, Fermi, no artigo sobre a teoria dos raios- $\beta$, coloca como uma problemática o "elétron nuclear", se referindo que, em acordo com a Teoria da Relatividade, é difícil explicar o elétron ligado em uma órbita de dimensões nucleares [13, p. 1].

Diante desse contexto, uma visão interessante a respeito dos prótons e nêutrons, que auxilia na compreensão do tema aqui abordado, é fornecido por Heisenberg, o qual diz que os nêutrons e prótons podem ser considerados como dois estados quânticos internos diferentes de uma partícula pesada [14, p. 23]. Já Fermi, na sua teoria para o decaimento beta, usa esta formulação para explicar a transição de um nêutron em um próton acompanhada da criação de um elétron e um neutrino [13, p. 2], sendo também válida para a transição de um próton em um nêutron. Nessa mesma publicação, Fermi usou a analogia com a emissão de um quantum de luz (fóton) por um átomo excitado, para explicar a emissão de partículas leves (elétron e neutrino) pelo núcleo excitado. E, assim, como no caso dos fótons que são criados somente no ato de sua emissão pelo átomo, os elétrons e neutrinos são criados somente no momento da sua emissão pelo núcleo.

A desintegração beta foi a base das refutações para a existência do elétron no núcleo. Mas há outro processo já mencionado, o da captura eletrônica $(\varepsilon)$, que também pode ser enquadrado nestas considerações. Na captura eletrônica, um elétron é capturado das camadas mais internas da eletrosfera, geralmente a camada $K$. Pelas mesmas razões supracitadas, o elétron não vai existir no núcleo, porque no ato da sua captura há a conversão (transmutação) de um próton num nêutron e mais um neutrino, que também é criado no mesmo instante da captura e é emitido pelo núcleo.

\section{Agradecimentos}

Agradeço aos colegas Marcos Luís Cassal e Virginia Cielo Rech pela leitura crítica e sugestões incorporadas ao texto e, em especial, pelas valiosas discussões sobre o tema. Agradeço também o apoio do Centro Universitário Franciscano (Santa Maria/RS www.unifra.br), no Programa de Pós-graduação em Ensino de Ciências e Matemática (PPGECIMAT), pelas horas de pesquisa que permitiram a elaboração deste trabalho.

\section{Referências}

[1] H.A. Bethe e R.F. Bacher, Reviews of Modern Physics, 8, 82 (1936).

[2] A. Das e T. Ferbel, Introduction to Nuclear and Particle Physics. (John Wiley \& Sons, Inc., New York, 1994).

[3] R. Eisberg e R. Resnick, Física Quântica. (Campus, Rio de Janeiro, 1985).

[4] K. Heyde, Basic Ideas and Concepts in Nuclear Physics: An Introductory Approach. (CRC Press, London, 2004), 3rd ed.

[5] E. Rutherford, The London, Edinburgh, and Dublin Philosophical Magazine and Journal of Science, 4, 580 (1927).

[6] G. Gamow, Zeitschrift für Physik, 51, 204 (1928).

[7] F. Reif, Fundamentals of Statistical and Thermal Physics. (Waveland Press, Inc., Illinois, 2008).

[8] NuDat (Nuclear Structure and Decay Data), disponível em http://www.nndc.bnl.gov/nudat2/, acesso em 1/8/2016.

[9] K.C. Chuang, Introdução à Física Nuclear. (Ed UERJ, Rio de Janeiro, 2001).

[10] I. Angeli and K.P. Marinova, Atomic Data and Nuclear Data Tables 99, 69 (2013).

[11] L.D. Broglie, Recherches sur la Théorie des Quanta. Theses, Français, 1924, disponível em https://tel. archives-ouvertes.fr/tel-00006807, acesso em $31 / 10 / 2016$.

[12] P.J. Mohr, B.N. Taylor e D.B. Newelll, Reviews of Modern Physics 80, 633 (2008).

[13] E. Fermi, Il Nuovo Cimento 11, 1 (1934).

[14] W. Heisenberg, Zeitschrift fúr Physik 77, 1 (1932). 